\title{
Ocorrência de leucoencefalomalácia (LEME) em equídeos no estado de São Paulo, Brasil: achados anatomopatológicos
}

\author{
Occurrence of leukoencephalomalacia in equids in São Paulo state, Brazil: \\ anatomopathological findings
}

\begin{abstract}
Cláudia DEL FAVA ${ }^{1}$; Maria do Carmo Custódio Souza Hunold LARA ${ }^{1}$; Eliana Monteforte Cassaro VILLALOBOS $^{1}$; Alessandra Figueiredo de Castro NASSAR ${ }^{1}$; Aline Diniz CABRAL ${ }^{1}$; Camila Souza TORELLI $^{1}$; Mariana Sequetin CUNHA ${ }^{1}$; Elenice Maria Sequetin CUNHA ${ }^{1}$
\end{abstract}

${ }^{1}$ Centro de Pesquisa e Desenvolvimento de Sanidade Animal do Instituto Biológico, São Paulo-SP

\begin{abstract}
Resumo
O trabalho relata a ocorrência de leucoencefalomalácia em equídeos (LEME) com sintomatologia nervosa e com diagnóstico negativo para raiva, herpesvírus equino e encefalomielite equina durante o período de dois anos, no Estado de São Paulo, Brasil. Foram examinadas 67 amostras de sistema nervoso central e em 10,4\% (cinco equinos, um pônei e um asinino) observaram-se lesões macroscópicas de LEME, confirmadas pela análise histopatológica. Os animais acometidos eram cinco machos e duas fêmeas, com idades que variavam de 11 meses a nove anos. Os sete casos ocorreram tanto no inverno como em outras estações do ano. As principais manifestações clínicas relatadas foram incoordenação, ataxia, paralisia dos membros posteriores, profunda depressão, levando ao óbito. Macroscopicamente, observaram-se congestão dos vasos meníngeos, áreas de malácia da substância branca, caracterizadas por coloração amarelada e/ou hemorrágica, com cavitação e amolecimento circundados por hiperemia. As lesões microscópicas observadas em todos os casos eram de necrose de liquefação da substância branca do cérebro, caracterizada por substância eosinofílica amorfa e homogênea, presença de edema axonal e perivascular, hemorragia e vacuolização do neurópilo adjacente e esferoides axonais. Em algumas áreas de malácia havia também células Gitter. Em apenas um animal observou-se manguito perivascular mononuclear. O presente trabalho confirma que o diagnóstico diferencial é importante na distinção da LEME com outras neuropatias encefálicas que acometem equídeos. A ocorrência da LEME relatada neste estudo demonstra que esta enfermidade é importante para a equideocultura do Estado de São Paulo.
\end{abstract}

Palavras-chave: Leucoencefalomalácia. Equídeos. Diagnóstico diferencial. Macroscopia. Histopatologia.

\begin{abstract}
This article describes clinical and pathological findings of leukoencephalomalacia in equids with neurological signs which tested negative to rabies, equine herpesvirus and equine encephalomyelitis. This work was carried during the period of two years in São Paulo State, Brazil. A total of 67 brain samples were examined and in 10.4\% (five equines, one poney, and one donkey) were observed gross lesions of ELEM, confirmed by histopathological analysis. The animals were five males and two females ranged from 11 months to nine years old. The seven cases happened in all seasons of the year. The most characteristic clinical signs were incoordination, ataxia, paralysis of the hind legs, profound depression and death. Necropsy was performed to collect brain samples for virological and histopathological diagnosis. Gross lesions included congestion of meningeal blood vessels, malacia of the white matter characterized by yellowish depressed areas sometimes hemorrhagic, with cavitations, and softening surrounded by hyperemic area. Microscopically, the lesions were liquefactive necrosis of the white matter brain, characterized by eosinophilic and amorphous material, axonal and perivascular edema, hemorrhage and vacuolization of the neuropil and axonal sferoids. Gitter cells were seen in some areas of malacia. Perivascular mononuclear cuffing was observed in only one case. The present study confirms that differential diagnosis is very important to distinguish equid neuropathies. The occurence of ELEM in the present study shows that the disease is important for the equideoculture in São Paulo State.
\end{abstract}

Keywords: Leukoencephalomalacia. Equids. Diferencial diagnosis. Gross lesions. Histopathology.

\section{Introdução}

A Leucoencefalomalácia Equina (LEME) é uma doença causada pela micotoxina fumonisina $B_{1}$, um metabólito do fungo Fusarium moliniforme ${ }^{1}$, uma espécie bem adaptada a climas tropicais ${ }^{2}$. A doença frequentemente ocorre pela ingestão de milho contaminado pelo F. moliniforme, mas também pode estar
Correspondence to:

Elenice Maria Sequetin Cunha

Centro de Pesquisa e Desenvolvimento de Sanidade Animal

Laboratório de Raiva e Encefalites Virais

Instituto Biológico

Av. Cons. Rodrigues Alves, 1252

CEP-04014-002, São Paulo- SP

E-mail:cunha@biologico.sp.gov.br

Recebido: 08/09/2009

Aprovado: 09/12/2010 
associada ao consumo de rações comerciais ${ }^{3}$. Casos da doença já foram descritos em diversas regiões do Brasil e geralmente estão associados à ingestão de milho contaminado ${ }^{4,5,6,7,8,9,10}$.

O curso clínico da doença está diretamente relacionado à quantidade de toxina ingerida, sendo influenciado pela tolerância individual. A evolução clínica da LEME é geralmente aguda e os sinais são observados em algumas horas ou até vários dias após o consumo de alimento contaminado, mas geralmente o diagnóstico é realizado em um período de 72 horas $^{4}$.

Os sinais clínicos mais frequentes são fraqueza generalizada; hiperexcitabilidade ou estado mental semicomatoso; acuidade visual ausente uni ou bilateral; desorientação; ataxia, andar em círculos; pressão da cabeça contra objetos; fasciculações musculares; ptose auricular, palpebral e labial, provocando dificuldade na apreensão de alimentos e hipoalgesia da face. Alguns animais doentes exibem comportamento frenético, correndo de forma desesperada, podendo posteriormente permanecer em decúbito, apresentar convulsões tônico-clônicas, coma e morte ${ }^{11}$.

As enfermidades que afetam o sistema nervoso de equinos muitas vezes apresentam sintomatologia clínica semelhante sendo, portanto, confundíveis. Entre estas, pode-se citar a raiva, a mieloncefalite herpética, as encefalites americanas, o tétano, os nematoides cerebrais, a listeriose, a mieloencefalite por protozoários, entre outras. Por este motivo o diagnóstico diferencial é indicado ${ }^{7}$.

O objetivo do presente trabalho foi relatar a ocorrência de leucoencefalomalácia em equídeos com sintomatologia nervosa e com diagnóstico negativo para raiva, herpesvírus equino e encefalomielite equina.

\section{Material e Método}

Foram examinadas, de junho de 2007 a junho de 2009, 67 amostras de sistema nervoso central de equídeos, com manifestações clínicas de distúrbios nervosos ou alterações comportamentais, colhidas por médicos veterinários autônomos e da Coordenadoria de Defesa Sanitária do Estado de São Paulo. As amostras foram enviadas ao laboratório acondicionadas em gelo ou gelo/formol. Aquelas negativas para raiva e que foram enviadas conservadas sob refrigeração foram fixadas em formol tamponado $10 \%$. Os fragmentos eram principalmente de córtex cerebral e de cerebelo. Foram realizados cortes das amostras, buscando-se incluir no fragmento que seria processado histologicamente as áreas com alterações macroscópicas de cor e consistência. O processamento histotécnico das amostras de SNC seguiu o protocolo preconizado pelo Ministério da Agricultura, Pecuária e Abastecimento ${ }^{12}$.

\section{Resultados}

Das 67 amostras analisadas, 10,4\% ( $\mathrm{n}=7)$ apresentaram lesões macroscópicas de LEME, confirmadas pela análise histopatológica. Somente em um dos casos foi informado que o animal recebia suplementação alimentar com milho e quirera. Os dados sobre a espécie, idade, raça e sexo dos animais, período de evolução clínica e mês da ocorrência, informados pelos veterinários remetentes estão descritos no quadro 1 . Os principais sintomas clínicos relatados em algumas das fichas encaminhadas com as amostras relatavam sintomas neurológicos como incoordenação, ataxia e paralisia dos membros posteriores.

As lesões macroscópicas puderam ser observadas nas amostras que foram enviadas conservadas sob refrigeração (Quadro 2). Em todos os casos observouse congestão dos vasos meníngeos, áreas de malácia da substância branca caracterizada por coloração amarelada e ou hemorrágica, com cavitação e amolecimento circundados por hiperemia. Quanto à localização anatômica, em todos os materiais havia lesões na substância branca do córtex cerebral e em apenas dois casos observou-se também malácia no tálamo. 


\begin{tabular}{|c|c|c|c|c|c|c|c|}
\hline Caso & Município & Espécie & Raça & Sexo & Idade & $\begin{array}{c}\text { Evolução } \\
\text { clínica }\end{array}$ & $\begin{array}{c}\text { Mês/ano } \\
\text { ocorrência }\end{array}$ \\
\hline 01 & Monte Mor & Equina & Mangalarga Paulista & $\mathrm{F}$ & adulta & - & Junho/07 \\
\hline 02 & Indaiatuba & Equina & - & $\mathrm{M}$ & 1 ano & - & Agosto/07 \\
\hline 03 & Águas de Lindoia & Equina & Pônei - SRD & $\mathrm{M}$ & 6 anos & 2 dias & Agosto/07 \\
\hline 04 & Indaiatuba & Equina & Mangalarga marchador & $\mathrm{M}$ & 11 meses & 3 dias & Outubro/07 \\
\hline 05 & Não informado & Equina & - & $\mathrm{F}$ & - & - & Agosto/08 \\
\hline 06 & Monte Sião & Asinina & Pêga & $\mathrm{M}$ & 9 anos & 12 dias & Janeiro/09 \\
\hline 07 & Oscar Bressane & Equina & - & $\mathrm{M}$ & 5 anos & 3 dias & Abril/09 \\
\hline
\end{tabular}

Quadro 1 - Casos de LEME ocorridos no Estado de São Paulo, segundo o município, a espécie, raça, sexo, idade, tempo de evolução clínica e mês/ano da ocorrência - São Paulo - 2009

\begin{tabular}{|c|c|l|}
\hline Caso & Espécie & \multicolumn{1}{|c|}{ Localização anatômica e alterações microscópicas } \\
\hline 01 & Equina & $\begin{array}{l}\text { No fragmento de tálamo e tronco encefálico, observou-se ao redor da área de malácia congestão do neurópilo, } \\
\text { edema perivascular e perineuronal, bem como a presença de esferoides, edema e necrose axonal. O córtex } \\
\text { cerebral apresentou intenso edema e congestão tanto da substância branca quanto cinzenta. }\end{array}$ \\
\hline 02 & Equina & $\begin{array}{l}\text { Observou-se à macroscopia grande área de cavitação na substância branca de um fragmento de córtex cerebral; } \\
\text { o histopatológico revelou ao redor da área de cavitação congestão vascular, com manguito perivascular } \\
\text { mononuclear, gliose, edema axonal, presença de células Gitter. }\end{array}$ \\
\hline 03 & Equina & $\begin{array}{l}\text { A histopatologia revelou em um fragmento de SNC hemorragia, edema e congestão na substância branca, } \\
\text { presença de células Gitter. }\end{array}$ \\
\hline 04 & Equina & $\begin{array}{l}\text { A macroscopia observou-se diversas áreas da substância branca da córtex cerebral e do tronco encefálico } \\
\text { necrose de liquefação. Observou-se à microscopia que, ao redor da área de malácia havia intensa congestão } \\
\text { vascular, edema, degeneração e necrose axonal, presença de células Gitter. }\end{array}$ \\
\hline 05 & Equina & $\begin{array}{l}\text { A macroscopia indicou, em um dos hemisférios cerebrais, malácia e congestão ao redor desta área. Na } \\
\text { microscopia observou-se intensa congestão vascular e presença de células Gitter. }\end{array}$ \\
\hline 06 & Asinina & $\begin{array}{l}\text { A macroscopia revelou, no tálamo, uma área de malácia. A microscopia revelou, nessa área, edema e } \\
\text { degeneração axonal, intensa congestão vascular ao redor da área de malácia e gliose, degeneração e tumefação } \\
\text { de axônios. Na substância cinzenta havia acentuado edema e congestão vascular. }\end{array}$ \\
\hline 07 & Equina & $\begin{array}{l}\text { A macroscopia revelou, no tálamo, área de malácia. A microscopia revelou, nesta área, esferoides, edema e } \\
\text { necrose axonal, com células Gitter. Havia intensa congestão vascular ao redor da área de malácia, gliose e } \\
\text { discreto manguito perivascular mononuclear. }\end{array}$ \\
\hline
\end{tabular}

Quadro 2 - Localização anatômica e alterações microscópicas observadas no SNC de equídeos acometidos por LEME no Estado de São Paulo, segundo a espécie - São Paulo - 2009

As lesões microscópicas observadas em todos os casos (Quadro 2) foram semelhantes. A necrose de liquefação da substância branca do cérebro apresentava-se com coloração eosinofílica, aspecto amorfo e homogêneo. $\mathrm{O}$ neurópilo circunjacente à malácia apresentava-se congesto e vacuolizado, com edema perivascular e axonal, bem como havia inúmeros esferoides axonais e gliose. Em algumas áreas de necrose havia também macrófagos espumosos (células Gitter). Em apenas um animal observou-se moderado manguito perivascular mononuclear.

\section{Discussão}

O diagnóstico clínico conclusivo de LEME é difícil de ser realizado, pois os sinais neurológicos são semelhantes àqueles que ocorrem em decorrência da raiva, da encefalopatia por herpesvírus e das encefalomielites. Nem sempre as lesões patognomônicas de leucoencefalomalácia são observadas macroscopicamente, podendo haver múltiplos e pequenos focos vermelhos de hemorragia distribuídos aleatoriamente na substância branca ${ }^{13}$, portanto, o diagnóstico anatomopatológico da LEME deve ser baseado tanto nas 
alterações macroscópicas quanto microscópicas do sistema nervoso central.

Os achados no presente estudo indicam que a leucoencefalomalácia é uma doença importante para equídeos sendo causa da morte de $10,4 \%$ dos animais estudados. O diagnóstico da LEME foi baseado nas manifestações clínicas características de acometimento cerebral, alterações macroscópicas e histológicas características. Cabe ressaltar que não somente a coleta do SNC, mas também as informações clínicas, epidemiológicas e de necropsia foram cedidas pelos veterinários autônomos e oficiais que enviaram as amostras para análise laboratorial. Nem sempre todas as porções anatômicas do SNC foram enviadas para diagnóstico, sendo assim impossível definir com exatidão a localização anatômica macroscópica das lesões, embora em todos os materiais tenham sido observadas áreas de malácia na substância branca do córtex cerebral (Quadro 2). Em apenas três casos (equinos 01 e 07; asinino 06) as mesmas lesões foram detectadas também no tálamo (Quadro 2). Diversos autores que relataram a LEME em equídeos no Brasil verificaram que as lesões macroscópicas localizam-se principalmente na substância branca dos hemisférios cerebrais e menos frequentemente no tronco encefáli$\operatorname{co}^{7,11,13,14}$, comprovando que as lesões de necrose de liquefação da substância branca de um ou dos dois hemisférios cerebrais são patognomônicas da enfermidade $^{15}$. Os achados neste estudo também concluíram que as lesões foram mais frequentemente observadas na substância branca do córtex cerebral.

Com relação aos achados histopatológicos, as lesões de necrose da substância branca são condizentes com as relatadas por outros autores ${ }^{7,11,13,14}$.

A LEME já foi diagnosticada em cavalos de diversas regiões do Brasil ${ }^{5,6,8,13,16,17}$, sendo relatada em muares em Pernambuco ${ }^{11}$ e outro no Pará ${ }^{18}$.

O presente trabalho relata a ocorrência de LEME em animais do Estado de São Paulo em um período de dois anos. Dentre os sete animais acometidos e descritos neste estudo havia cinco equinos, um pônei e um asinino. A LEME poder acometer asininos, no entanto são poucos os dados da literatura referentes à sua ocorrência nessa espécie ${ }^{15,19}$, tendo sido descrito recentemente um surto da doença no México ${ }^{20}$. Porém, não há dados sobre a ocorrência da doença em asinino no Brasil, sendo o relato deste trabalho o primeiro caso descrito. Outros estudos devem ser realizados para que se avalie uma possível resistência desta espécie à fumonisina.

Dentre os sete animais doentes, cinco eram machos e dois eram fêmeas, com idades que variavam entre 11 meses a nove anos. A ocorrência da LEME, segundo vários autores, está relacionada à idade do animal, e os adultos são mais acometidos ${ }^{21,22}$. No presente trabalho nem todos os animais diagnosticados eram adultos. Câmara et al. ${ }^{11}$ também descreveram a doença em equino jovem e comentaram que a maior ocorrência da doença em animais adultos pode estar relacionada ao tipo de alimentação fornecida aos animais jovens, que é menos rica em milho e outros concentrados.

Em relação à evolução clínica, os animais vieram a óbito em um período que variou de dois a 12 dias. Outros autores também relatam que a LEME pode causar a morte dos animais em um período que pode variar de horas até dias ${ }^{11,22,23}$.

Como ilustrado na Quadro 1, os casos relatados ocorreram tanto no inverno como em outras estações do ano. Segundo Méndez e Riet-Correa ${ }^{24}$, nas regiões sul e sudeste a doença é sazonal e ocorre principalmente no inverno entre os meses de junho e setembro, quando os animais, por falta de pastagem, recebem maior quantidade de milho ou subprodutos. Os achados neste estudo corroboram em parte com esta afirmação, já que $57 \%$ dos casos ocorreram no inverno.

A ocorrência da LEME descrita no presente estudo sugere que equídeos do Estado de São Paulo recebem alimentos contaminados com fumonisina $\mathrm{B}_{1}$ e que medidas de prevenção devem ser adotadas, recomendando-se que o milho ou seus subprodutos não sejam ad- 
ministrados em quantidades superiores a $20 \%$ do total da matéria seca ingerida, e que se utilize milho que tenha sido submetido a um correto processo de secagem e armazenado em local livre de umidade ${ }^{11,22,24}$.

\section{Conclusões}

O diagnóstico diferencial realizado em amostras de SNC enviadas com suspeita de raiva foi importante na distinção da LEME com outras neuropatias encefálicas que acometem equídeos. A ocorrência da LEME

\section{Referências}

1.SHEPARD, G. S.; THIEL, P. G.; SYDENHAM, E. W. Initial studies on the toxicokinetics of fumonisin $\mathrm{B}$ in rats. Food and Chemical Toxicology, v. 30, p. 277-279, 1992

2.SYDENHAM, E. W.; GELDERBLOM, W. C. A.; THIEL, P. G.; MARASAS, W. F. O. Evidence for the natural occurrence of fumonisin $\mathrm{B}_{1}$, a mycotoxin produced by Fusarium moniliforme, in corn. Journal of Agriculture Food Chemical, v. 38, p. 285290, 1990.

3. WILSON, T. M.; NELSON, P. E.; RYAN, T. B. Linking leucoencephalomalacia to commercial horse rations. Veterinary Medicine, v. 80, p. 63-69, 1985.

4. MALLMANN, C. A.; SANTURIO, J. M.; DILKIN, P. Leucoencefalomalácia equina associada à ingestão de milho contaminado por fumonisina $B_{1}$. Revista de Microbiologia, v. 30, n. 3, p. 249-252, 1999.

5.DANTAS, A. F. M.; GIRARDI, N.; SCHEIN, F. B.; GRECCO, F. B.; COLVARA, I. G.; ÁVILA, M. O. Leucoencefalomalácia em equinos no Estado de Mato Grosso. In: ENCONTRO NACIONAL DE PATOLOGIA VETERINÁRIA, 10, 2001, Pirassununga. Anais... Pirassununga, 2001. p. 155.

6. MEIRELES, M. A.; CORRÊA, B.; FISCHMAN, O.; GAMBALE, W.; PAULA, C. R.; CHACON-RECHE, N. O.; POZZI, C. R. Mycoflora of the toxic feeds associated with equine leucoencephalomalacia (ELEM) outbreaks in Brazil. Mycopathology, v. 127, n. 1-2, p. 183-188, 1994.

7.RIET-CORREA, F.; MEIRELLES, M. A.; SOARES, J. M.; MACHADO, J. J.; ZAMBRANO, A. F. Leucoencefalomalácia em equinos associada à ingestão de milho mofado. Pesquisa Veterinária Brasileira, v. 2, n. 1, p. 27-30, 1982.

8. RIET-CORREA，F.; TABOSA， I. M.; AZEVEDO, E. O.; MEDEIROS, R. M. T.; SIMÕES, S. V. D.; DANTAS, A. A.; ALVES, J. C.; NOBRE, V. M. T.; ATHAYLDE, A. C.; GOMES, A. A.; LIMA, E. F. Doenças de ruminantes e equinos no semi-árido da Paraíba. Semi-Árido em Foco, v. 1, p. 2-86, 2003.

9. RIET-CORREA， G.; DUARTE， M. D.; CERQUEIRA, V. D.; RIET-CORREA, F. Leucoencephalomalacia in mules in Northern Brazil. In: PANTER, K. E.; WIERENGA, T. L.; PFISTER, J. A. Poisonous plants: global research and solutions. Wallingford, Oxon, UK: CABI Publishing, 2007. p. 256-262.

10.PIMENTEL, L. A.; OLIVEIRA, D. M.; GALIZA, G. J. N.; REGO, R. O.; DANTAS, A. F. M.; RIET-CORREA, F. Doenças relatada neste estudo demonstra que esta enfermidade é importante para a equideocultura do Estado de São Paulo.

\section{Agradecimentos}

À Fundação de Amparo a Pesquisa do Estado de São Paulo (FAPESP) e ao Conselho Nacional de Desenvolvimento Científico e Tecnológico/Ministério da Agricultura Pecuária e Abastecimento (CNPq/ MAPA) pelo apoio financeiro.

do sistema nervoso central de equídeos no semi-árido. Pesquisa Veterinária Brasileira, v. 29, n. 7, p. 589-597, 2009.

11.CÂMARA, A. C. L.; AFONSO, J. A. B.; RIET-CORREA, F.; DANTAS, A. F. M.; MENDONCA, C. L.; COSTA, N. A.; DANTAS, A. C.; COSTA NETO, H. A.; CAMPOS, A. G. S. S., SOUZA, M. I. Leucoencefalomalácia em equídeos no Estado de Pernambuco. Ciência Animal Brasileira, v. 9, n. 2, p. 470479, 2008.

12.BARROS, C. S. L.; MARQUES, G. H. F. Procedimentos para o diagnóstico das doenças do sistema nervoso central de bovinos. Brasília: MAPA/DSA/DDA, 2003. 50 p.

13. BARROS, C. S. L.; BARROS, S. S.; SANTOS, M. N.; SOUZA, M. A. Leucoencefalomalácia em equinos no Rio Grande do Sul. Pesquisa Veterinária Brasileira, v. 4, n. 3, p. 101-107, 1984

14.RABELO, J. H. O.; RIET-CORREA, F; BARROS, C. S. L.; DANTAS, A. F. M.; SCHILD, A. L.; TABOSA, I. M. Distribuição das lesões macroscópicas em 15 casos de leucoencefalomalácia dos equinos. Pesquisa Veterinária Brasileira, v. 24, p. 46-47, 2004. Suplemento.

15.WILSON, B. G.; MARONPOT, R. R. Causative fungus agent of leukoencephalomalacia in equine animals. Veterinary Record, v. 88, p. 484-486, 1971.

16. HIROOKA, E. Y.; VIOTTI, N. M. A.; SOARES, L. M. V.; ALFIERI, A. L. Intoxicação em equinos por micotoxinas produzidas por Fusarium moniliforme no norte do Paraná. Semina, v. 9, p. 128-135, 1988.

17. REGO CHAVES, L. Doença de sintomatologia nervosa causada pela intoxicação pelo milho. Revista Medicina Veterinária, v. 10, p. 199-207, 1950.

18. RIET-CORREA, G.; DUARTE, M. D.; CERQUEIRA, V. D.; RIET-CORREA, F. Leucoencephalomalacia in mules in Northern Brazil. In: PANTER, K. E.; WIERENGA, T. L.; PFISTER, J. A. Poisonous plants: global research and solutions. Wallingford, Oxon, UK: CABI Publishing, 2007. p. 256-262.

19.BADIALI, L.; ABOU-YOUSSEF, M. H.; RADWAN, A. I.; HAMDY, F. M.; HILDEBRANDT, P. K. Moldy corn as the major cause of an encephalomalacia syndrome in Egyptian equidae. American Journal of Veterinary Research, v. 29, p. 2029-2035, 1968.

20.ROSILES, M. R.; BAUTISTA, J.; FUENTES, V. O.; ROSS, F. An outbreak of equine leukoencephalomalacia at Oaxaca, Mexico, associated with fumonisin $\mathrm{B}_{1}$. Zentralblatt für 